\title{
Las habilidades intelectuales y el aprendizaje estratégico en estudiantes de estudios generales de una Universidad Privada de Lima
}

\author{
Carlos Alberto Peña Miranda ${ }^{1}$ y Elizabeth Cosi Cruz ${ }^{2}$
}

Resumen: El objetivo de la presente investigación fue establecer las correlaciones entre las habilidades intelectuales y el aprendizaje estratégico en una muestra de estudiantes de estudios generales de una Universidad Privada de Lima. El diseño de investigación utilizado es el no experimental de tipo correlacional y se trabajó con una muestra compuesta por 348 sujetos, a quienes se les aplicó el Inventario de Habilidades intelectuales de Rosario Lajo y el Inventario de Aprendizaje estratégico de Manuel Torres. Los resultados estadísticos alcanzados muestran la existencia de correlaciones significativas y positivas entre ambas variables $(\mathrm{r}=0,77)$, lo que permitió la aceptación de la primera hipótesis de investigación. Igualmente los resultados muestran la existencia de relaciones positivas y significativas de todas las dimensiones de las variables; habilidades intelectuales y aprendizaje estratégico, por lo que se aceptaron todas las hipótesis en estudio.

Palabras clave: habilidades intelectuales; aprendizaje estratégico; aprendizaje significativo.

\section{Intellectual skills and strategic learning in students of general studies of a Private University of Lima}

\begin{abstract}
The objective of this research was to establish the correlations between the Intellectual Skills and the Strategic Learning in a sample of students of General Studies from a Private University of Lima. The research design used is the nonexperimental of a correlational type. A sample of 348 subjects was used, to whom the Inventory of Intellectual Skills of Rosario Lajo and the Inventory of Strategic Learning of Manuel Torres were applied. The statistical results obtained show the existence of significant and positive correlations between both variables $(\mathrm{r}=0,77)$, which allowed the acceptance of the first research hypothesis. Moreover, the results show the existence of positive and significant relations of all the dimensions of the variables, intellectual skills and strategic learning; therefore, all the studied hypotheses were accepted.
\end{abstract}

Keywords: intellectual skills; strategic learning; significant learning.

Recibido: 23/12/2018. Aceptado: 14/06/2019. Publicado online: 26/12/2019.

(C)Los autores. Este artículo es publicado por la Revista PESQUIMAT de la Facultad de Ciencias Matemáticas, Universidad Nacional Mayor de San Marcos. Este es un artículo de acceso abierto, distribuido bajo los términos de la licencia Creative Commons Atribucion-No yor de San Marcos. Este es un artículo de acceso abierto, distribuido bajo los términos de la licencia Creative Commons Atribucion-No
Comercia-Compartir Igual 4.0 Internacional.(http://creativecommons.org/licenses/by-nc-sa/4.0/) que permite el uso no comercial, distribución y reproducción en cualquier medio, siempre que la obra original sea debidamente citada. Para información, por favor póngase en contacto con revistapesquimat.matematica@unmsm.edu.pe

\footnotetext{
${ }^{1}$ UNMSM, Facultad de Ciencias Matemáticas. e-mail: cpenam@unmsm.edu.pe

${ }^{2}$ Universidad Privada Norbert Wiener, e-mail: elizabeth.cosi@uwiener.edu.pe
} 


\section{Introducción}

El desarrollo actual de las sociedades, está signada por el cambio continuo y veloz, tanto en la ciencia como en la tecnología, lo que ha llevado a que estos cambios se manifiesten, también, en los sectores de la producción y prestación de servicios, lo que a su vez trae una nueva exigencia; la formación de profesionales convenientemente preparados para enfrentar las nuevas condiciones del desarrollo social y eso pasa, necesariamente, por desarrollar en ellos las habilidades y capacidades necesarias como para asumir y manejar el conocimiento, tal como lo señala Monereo (2007).

En los tiempos actuales resulta fundamental no la acumulación de conocimientos, sino el aprender a cómo apropiarse de los conocimientos, es decir, aprender a aprender. Es verdad que todos los estudiantes universitarios quieren y pueden convertirse en profesionales exitosos y reconocidos; su estructura cerebral y disposiciones hereditarias lo permiten, sin embargo no basta con ello, es necesario formar el pensamiento, las habilidades intelectuales, los estilos y estrategias de aprendizaje.

En este aspecto, las habilidades intelectuales les dan a los estudiantes la capacidad suficiente como para manejarse de manera correcta por el mundo teniendo claramente establecidos sus objetivos vitales, además de la madurez suficiente como para presentar diversidad de alternativas ante los diversos problemas que se presenten en el mundo a consecuencia de los permanentes cambios que se suceden. Sin embargo la realidad dista mucho de ser la óptima. Estudios realizados por Zilberstein y Silvestre (2005), muestran que el desarrollo de las habilidades intelectuales continua siendo un serio problema entre los estudiantes de los diversos niveles educativos y que se expresan en las deficiencias para resolver tareas como comparaciones, clasificaciones, poner ejemplos, valorar los diferentes eventos o situaciones, solucionar problemas, plantear hipótesis, pero también explicar, argumentar, definir, etc.

Son estas razones por las que los sistemas educativos deben buscar con afán la formación de las habilidades intelectuales pues ellas les darán a los estudiantes mejores posibilidades para poder asimilar y manejar los aprendizaje, lo que redundaran en una mejor formación profesional y un mejor desempeño de sus actividades académicas y posteriormente laborales. En este proceso, desarrollar competencias para el aprendizaje, que les permita aprender a aprender resulta fundamental en tanto la velocidad de los cambios en el terreno del conocimiento hace imposible que el docente pueda enseñar todo. El estudiante tiene que regular sus procesos de aprendizaje de tal manera que pueda orientarlos hacia los objetivos previamente trazados.

El aprender a aprender, las estrategias de aprendizaje, constituyen uno de los focos de investigación más relevante en lo que se refiere a materia educativa, Monereo (2007), pues ellas son fundamentales a la hora en que se debe producir la interiorización y posterior desarrollo de los procedimientos para adquirir conocimientos, retenerlos y utilizarlos. En este proceso resulta de especial importancia la enseñanza de estrategias de aprendizaje, pues ellas van a permitir manejar todo el proceso de aprendizaje desde su planificación hasta su evaluación. Se procura que los estudiantes manejen una serie de procedimientos o estrategias de aprendizaje a fin de que pueda llegar a autorregularlo de manera tal que se pueda convertir en un estudiante estratégico, reflexivo, autónomo, en suma un aprendiz estratégico capaz de autorregular sus aprendizajes.

Los alumnos de estudios generales de una Universidad Privada de Lima, quienes se encuentran al inicio de su vida académica, presentan dificultades para su adecuación al nuevo sistema de educación superior en la cual tienen que hacer uso de abundante manejo de contenidos conceptuales y procedimentales. Estas dificultades iníciales se deben corregir rápidamente o de lo contrario van a permanecer en el tiempo con las consecuencias que ello significa. El desconocimiento de herramientas de estudio como las estrategias cognitivas trae como consecuencia que 
los alumnos requieren más esfuerzo y tiempo para el conocimiento de una asignatura, generando tensiones, desaprobación, retiro de la asignatura o lo más grave abandono de la Universidad.

Por lo expuesto, el presente trabajo tuvo por objetivo describir los niveles de habilidades intelectuales que presentan los estudiantes de estudios generales de una Universidad Privada de Lima..

\section{Metodología}

En la presente investigación se utilizó el método hipotético-deductivo pues el investigador formula una hipótesis como tentativa de solución al problema en el que todos sus términos están operacionalmente definidos y diseña una observación controlada para recoger evidencia empírica, que le permita confirmar o rechazar la hipótesis. Si los datos confirman la hipótesis considera que ha establecido una relación funcional de un valor predictivo generalmente restringido (García Hoz, 1994).

\section{Diseño de investigación}

El diseño que se ha utilizado es el no experimental de tipo correlacional transversal, de acuerdo a lo señalado por Hernández, Fernández y Baptista (2014), en tanto su propósito es describir, pero no las variables individuales sino las relaciones entre ellas, sean estas puramente correlacionales o relaciones causales. En estos diseños lo que se mide es la relación entre variables en un tiempo determinado.

\section{Muestra}

La muestra utilizada en la presente investigación es probabilística de tipo estratificado clasificando a los estudiantes de acuerdo a la carrera profesional a la que ingresaron. Del total de la población (3690), se determinó una muestra representativa mediante la técnica de muestreo aleatorio y por afijación proporcional. Para la estimación del tamaño de la muestra se utilizó la fórmula de Sierra Bravo (2003), la que dio como resultado un total de 348 estudiantes.

Tabla 1. Distribución de la muestra por Ciclo Académico

\begin{tabular}{|c|c|c|}
\hline Ciclo & Frecuencia & Porcentaje \\
\hline I & 104 & 29,9 \\
\hline II & 89 & 25,6 \\
\hline III & 68 & 19,5 \\
\hline IV & 87 & 25,0 \\
\hline Total & 348 & 100,0 \\
\hline
\end{tabular}

\section{Instrumentos}

Los instrumentos de recolección de datos que se utilizaron en el desarrollo de la presente investigación fueron:

- Habilidades intelectuales: Inventario de Habilidades intelectuales de Rosario Lajo.

- Aprendizaje estratégico: Inventario de Aprendizaje estratégico de Manuel Torres. 


\section{Análisis de validez y confiabilidad de los instrumentos}

En la medida de que es necesario garantizar que los instrumentos de evaluación cuenten con la necesaria validez y confiabilidad, es que se efectuaron los análisis respectivos los que dieron como resultados que, en el caso del inventario de habilidades intelectuales se obtiene un alfa de Cronbach de 0,88 lo que indica que indica un consistente nivel de confiabilidad. En lo que se refiere al inventario de aprendizaje estratégico, el alfa de Cronbach alcanzado es de 0,96, por lo que confirma que esta prueba es altamente confiable.

En lo que se refiere a los análisis de validez de los instrumentos, estos se realizaron utilizando el Análisis factorial Exploratorio cuyos resultados nos muestran que ambas pruebas presentan validez de Constructo.

\section{Resultados}

\subsection{Análisis descriptivos}

Tabla 2. Test de Bondad de Ajuste a la Curva Normal de Kolmogorov - Smirnov de las Variables estudiadas

\begin{tabular}{|c|c|c|c|c|}
\hline Variable & Media & D. E. & K-SZ & Sig. \\
\hline H. Lingüística & 35,90 & 6,00 & 0,069 & 0,021 \\
\hline H. Matemática & 35,06 & 6,01 & 0,071 & 0,016 \\
\hline H. Espacial & 35,10 & 5,38 & 0,053 & 0,200 \\
\hline H. Corporal & 35,87 & 5,48 & 0,0749 & 0,004 \\
\hline H. Musical & 34,95 & 6,46 & 0,073 & 0,012 \\
\hline Motivación Intrínseca & 16,08 & 2,49 & 0,147 & 0,000 \\
\hline Motivación Extrínseca & 17,30 & 2,43 & 0,163 & 0,000 \\
\hline Valor de la Tarea & 16,93 & 2,43 & 0,141 & 0,000 \\
\hline Autoeficacia y expectativas & 15,75 & 2,58 & 0,095 & 0,000 \\
\hline Planificación & 14,28 & 2,53 & 0,114 & 0,000 \\
\hline Autoevaluación & 15,62 & 2,40 & 0,108 & 0,000 \\
\hline Control y autorregulación & 19,15 & 3,22 & 0,126 & 0,000 \\
\hline Control del Contexto & 15,08 & 2,81 & 0,133 & 0,000 \\
\hline Aprendizaje con Compañeros & 18,82 & 3,35 & 0,106 & 0,000 \\
\hline Selección de información & 15,01 & 2,44 & 0,107 & 0,000 \\
\hline Adquisición de información & 16,01 & 2,51 & 0,168 & 0,000 \\
\hline Elaboración & 19,44 & 3,04 & 0,142 & 0,000 \\
\hline Organización & 18,84 & 3,51 & 0,100 & 0,000 \\
\hline Personalización y creatividad & 19,34 & 3,02 & 0,124 & 0,000 \\
\hline${ }^{*} p<0,05^{* *} p<0,01^{* * *} p<0,001$ & & & \\
$N=348$ & & &
\end{tabular}

Como se puede observar en la tabla 2, los resultados del análisis de normalidad de las pruebas de habilidades intelectuales y aprendizaje estratégico, realizado con el test de bondad de ajuste a la curva normal de Kolmogorov-Smirnov, muestran que los coeficientes estadísticos son 
significativos, lo que demuestra la no existencia de distribución normal en las áreas por lo que es necesario la utilización de estadísticos de orden no paramétrico al momento de realizar los análisis de los datos a fin de probar con seguridad, las hipótesis planteadas.

Ademas se puede apreciar en la presente tabla 3 y figura 1 , los resultados nos muestran que los niveles de habilidades intelectuales de los estudiantes de la muestra, se ubican mayoritariamente en un nivel medio, 60,5\%, más atrás se encuentran el nivel alto con el 21,0\% y luego el nivel bajo con el $18,5 \%$.

Tabla 3. Niveles de Habilidades intelectuales

\begin{tabular}{|c|c|c|}
\hline Niveles & Frecuencias & Porcentajes \\
\hline Bajo & 64 & 18,5 \\
\hline Medio & 211 & 60,5 \\
\hline Alto & 73 & 21,0 \\
\hline Total & 348 & 100,0 \\
\hline
\end{tabular}

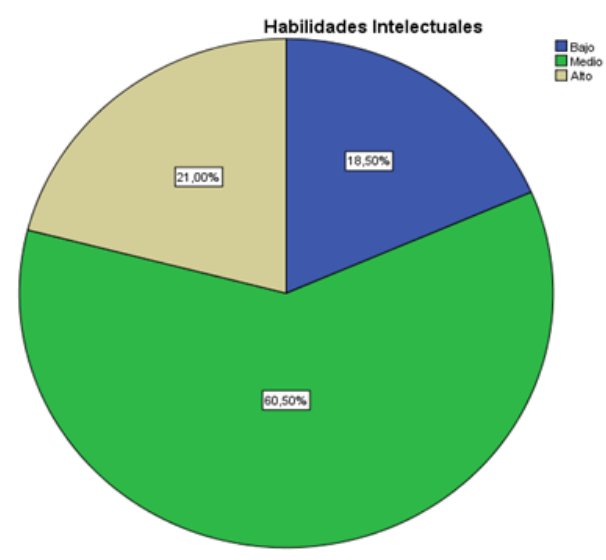

Figura 1. Niveles de habilidades intelectuales en los estudiantes de la muestra

Tal como se puede apreciar en la presente tabla 4 y figura 2, los resultados nos muestran que los niveles de aprendizaje estratégico de los estudiantes de la muestra, se ubican mayoritariamente en un nivel medio, 62,5\%, más atrás se encuentran el nivel alto con el 19,5\% y luego el nivel bajo con el 18,0\%.

Tabla 4. Niveles de Aprendizaje Estratégico

\begin{tabular}{|c|c|c|}
\hline Niveles & Frecuencias & Porcentajes \\
\hline Bajo & 63 & 18,0 \\
\hline Medio & 217 & 62,5 \\
\hline Alto & 68 & 19,5 \\
\hline Total & 348 & 100,0 \\
\hline
\end{tabular}




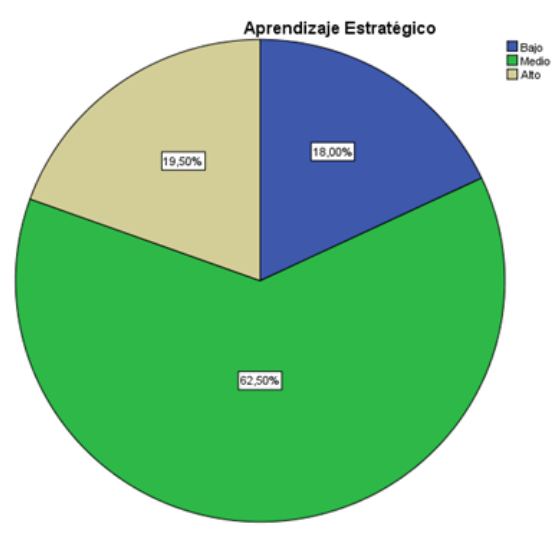

Figura 2. Niveles de aprendizaje estratégico en los estudiantes de la muestra

\subsection{Análisis Correlacionales descriptivos}

Como se puede observar en la Tabla 5 y figura 3 , los resultados nos indican que las habilidades intelectuales se encuentran relacionadas significativamente con el aprendizaje estratégico de los estudiantes.

Tabla 5. Análisis de Correlación (rho de Spearman) entre las habilidades intelectuales y el aprendizaje estratégico

\begin{tabular}{|c|c|}
\hline Variables & Aprendizajes estratégico \\
\hline Habilidades Intelectuales & $0,77^{* * *}$ \\
\hline
\end{tabular}

${ }^{*} p<0,05^{* *} p<0,01^{* * *} p<0,001$

$N=348$

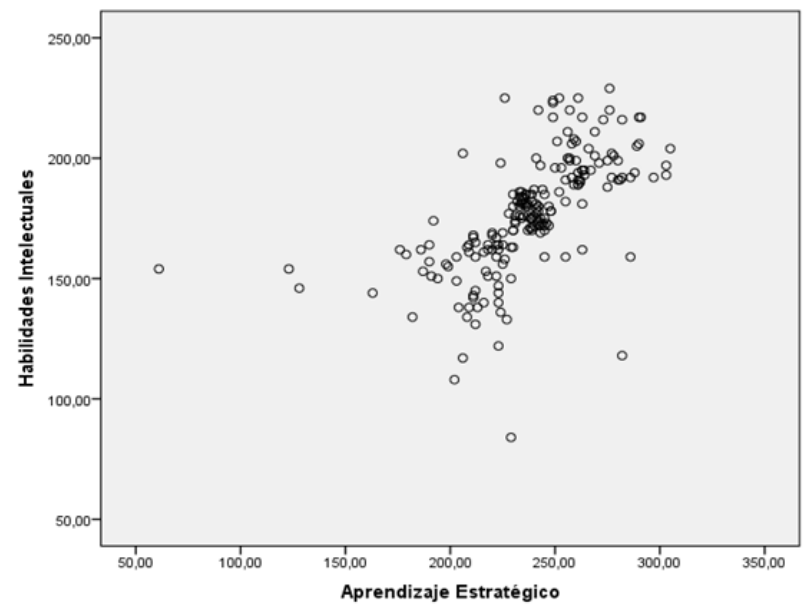

Figura 3. Correlación entre las habilidades intelectuales y el aprendizaje estratégico en los estudiantes de la muestra

Los resultados presentados en la Tabla 6 nos indican que, las dimensiones de ambas variables están correlacionadas significativamente entre sí en todos los casos. 


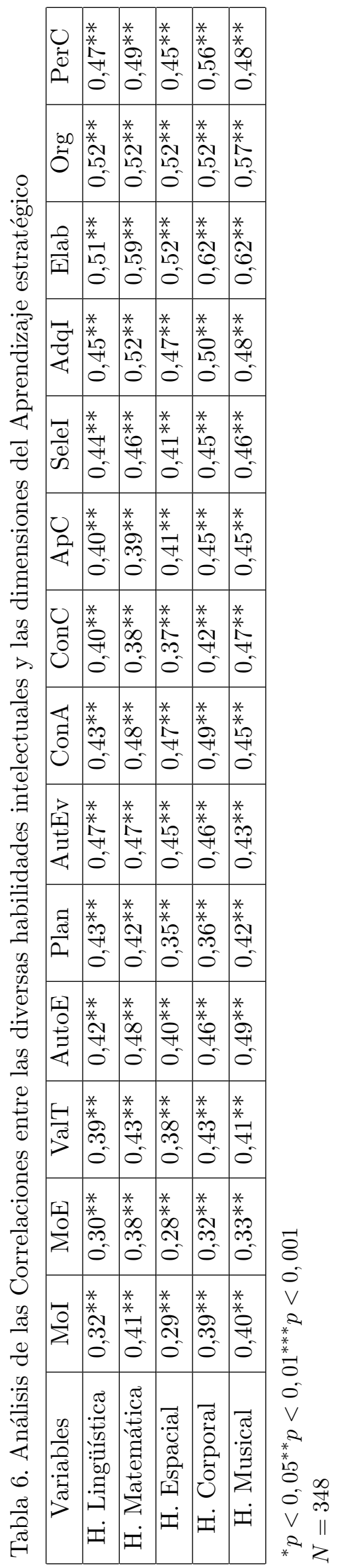


Los resultados presentados en la Tabla 7 nos indican que todas las diversas habilidades intelectuales se encuentran relacionadas significativamente con el aprendizaje estratégico de los estudiantes.

Tabla 7. Análisis de Correlación (rho de Spearman) entre las diversas habilidades intelectuales y el Aprendizaje estratégico

\begin{tabular}{|c|c|}
\hline Variables & Aprendizajes estratégico \\
\hline H. Lingüística & $0,58^{* * *}$ \\
\hline H. Matemática & $0,64^{* * *}$ \\
\hline H. Espacial & $0,57^{* * *}$ \\
\hline H. Corporal & $0,62^{* * *}$ \\
\hline H. Musical & $0,64^{* * *}$ \\
\hline${ }^{*} p<0,05^{* *} p<0,01^{* * *} p<0,001$ \\
$N=348$
\end{tabular}

\section{Discusión de los resultados}

Es una importante regla en la investigación que los instrumentos que se utilizan en un proceso de investigación deben contar con dos requisitos fundamentales: validez y confiabilidad, de lo contrario los datos que se obtengan serán puestos en duda, por lo menos, lo que invalidaría la investigación. Por estas razones es que en la presente investigación se procedió a efectuar los análisis pertinentes, los mismos que dieron como resultado que tanto el inventario de habilidades intelectuales como el inventario de aprendizaje estratégico, presentan validez y confiabilidad.

En lo que se refiere a la hipótesis planteada "Las habilidades intelectuales se relacionan significativamente con el aprendizaje estratégico en estudiantes de estudios generales de una Universidad Privada de Lima", los resultados obtenidos y que están consignados en la tabla 5, muestran con claridad que ambas variables están relacionadas de manera significativa entre sí $(\mathrm{r}=0,77)$, lo que permite respaldar la hipótesis formulada. Estos resultados concuerdan con los reportados en la investigación realizada por Del Valle (2012) y Kohler, J. (2009), quienes resaltan la importancia que tiene la inteligencia y las habilidades intelectuales para poder predecir tanto el rendimiento académico, como todos los procesos de aprendizaje por lo que es fundamental la formación académica que se les puede ofrecer y dar a los estudiantes, sin embargo, no es el único factor que se debe considerar. Existen otras variables como la motivación y los objetivos vitales, que no necesariamente se evalúan en las pruebas pero que sin embargo son altamente significativas en el proceso de aprendizaje de los estudiantes.

Es necesario considerar que en la medida de que las demandas y exigencias de las habilidades intelectuales en los últimos tiempo son significativamente mayores, hay necesidad de fomentarlas y desarrollarlas en toda la Universidad a fin de que los estudiantes estén en condiciones de realizar todo un proceso de reflexión sobre sus aprendizajes significativos. Bajo estas consideraciones es que las habilidades intelectuales se convierten en uno de los recursos centrales que le van a permitir a los sujetos desarrollar todas las competencias que sean necesarias para desempeñarse eficientemente en el mundo laboral, social y familiar. Es importante anotar que todas estas competencias lo deben llevar a un desarrollo independiente en todos los aspectos de la vida de relación y en la que se incluye el aprendizaje, que en todos los casos debe ser autónomo y estratégico. 
Si bien es cierto que el aprendizaje es un proceso intrapersonal e interpersonal de carácter social, cultural y disciplinar, Torre Puente, (2007), también es cierto que los docentes deben desarrollar en ellos la capacidad de aprender por sí mismo, pues esta capacidad se ha convertido en un requisito previo para vivir en el mundo actual. De acuerdo a Martínez, (2005), es necesario que para lograr la capacidad de aprender por sí mismo, es necesario desarrollar en los estudiantes la autonomía en su propio proceso de aprendizaje, fijando sus propios objetivos y vinculando los diversos problemas y sus formas de solución.

\section{Conclusiones}

1. Los resultados de la investigación muestran que los niveles de las habilidades intelectuales de los estudiantes de una Universidad Privada de Lima, se encuentran, fundamentalmente, ubicadas a nivel medio.

2. Los resultados de la investigación muestran que los niveles del aprendizaje estratégico de los estudiantes de una Universidad Privada de Lima, se encuentran, fundamentalmente, ubicadas a nivel medio.

3. Los resultados correlacionales, efectuados con el estadístico rho de Spearman, nos indican que existen relaciones significativas entre las habilidades intelectuales y aprendizaje estratégico, en los estudiantes de una Universidad Privada de Lima.

4. Los resultados correlacionales, efectuados con el estadístico rho de Spearman, nos indican que existen relaciones significativas entre las diversas dimensiones de las habilidades intelectuales y el aprendizaje estratégico, en los estudiantes de una Universidad Privada de Lima.

5. Los resultados correlacionales, efectuados con el estadístico rho de Spearman, nos indican que existen relaciones significativas entre las diversas dimensiones de las habilidades intelectuales y las diversas dimensiones del aprendizaje estratégico, en los estudiantes de una Universidad Privada de Lima.

\section{Referencias bibliográficas}

[1] Carbonell, G. (2015). El desarrollo de habilidades intelectuales, desde la química en la enseñanza de adultos (Tesis de maestría). Instituto pedagógico latinoamericano y caribeño, La Habana.

[2] Del Valle, M. (2012). Modelo de gestión académica basado en el desempeño docente y su relación con el rendimiento académico en institutos de educación superior. Universidad de Oriente.

[3] García-Hoz, V. (1994). Problemas y métodos de investigación en educación personalizada. Madrid, España: Ediciones Rialp.

[4] Hernández, R.; Fernández, C. y Baptista, P. (2014). Metodología de la Investigación. México: Ed Mc Graw Hill.

[5] Kohler, J. (2009). Rendimiento académico asociado a las habilidades intelectuales y las estrategias de aprendizaje de universitarios de Lima, Perú. I Congreso Internacional de Investigación y Práctica Profesional en Psicología XVI Jornadas de Investigación Quinto 
Encuentro de Investigadores en Psicología del MERCOSUR. Facultad de Psicología, Universidad de Buenos Aires, Buenos Aires.

[6] Martínez Fernández, J.R. (2005). Concepción del aprendizaje, metacognición y cambio conceptual en estudiantes universitarios de Psicología (Tesis doctoral). Universidad de Barcelona, España.

[7] Monereo, C. (2007). Hacia un nuevo paradigma del aprendizaje estratégico: el papel de la mediación social, del self y de las emociones. Electronic Journal of Research in Educational Psychology. Revisado el 21 de octubre de 2018. Recuperado: http://www.redalyc.org/articulo.oa?id=293121946003

[8] Sierra Bravo, R. (2003). Tesis doctorales y trabajos de investigación científica. Madrid, España: Ediciones Paraninfo S.A. .

[9] Torre, J.C. (2007). Una triple alianza para un aprendizaje universitario de calidad. Madrid, España: Universidad Pontificia icai icade Comillas Madrid.

[10] Zilberstein, J y Silvestre, M. (2005). Didáctica desarrolladora desde el Enfoque Histórico Cultural. México: Ediciones CEIDE. 